\title{
Abnormal skeletal muscle bioenergetics in familial hypertrophic cardiomyopathy
}

\author{
Campbell H Thompson, Graham J Kemp, Doris J Taylor, Michael Conway, \\ Bheeshma Rajagopalan, Annie O'Donoghue, Peter Styles, William J McKenna, \\ George K Radda
}

\begin{abstract}
Objective-To determine the skeletal muscle metabolic manifestations of familial hypertrophic cardiomyopathy.

Design-A case-control study

Setting ${ }^{-31} \mathbf{P}$ magnetic resonance spectroscopy of the calf muscle was performed on volunteers from a centre specialising in familial hypertrophic cardiomyopathy. Patients-Five patients with abnormal $\beta$ myosin heavy chain protein in cardiac and skeletal muscle and five patients with a troponin $T$ abnormality in cardiac muscle were compared with healthy controls. Results-High energy phosphate metabolism in vivo was examined in a non-invasive manner. In resting muscle, the $\beta$ myosin heavy chain group had a higher ratio of phosphocreatine to ATP concentration (4.51 (SD 0.17)) than either the troponin $T$ group $(3.88(0.42))$ or controls $(n=16 ; 4.04(0 \cdot 40))$. Exercise duration was reduced compared to controls, and during the fourth minute of exercise phosphocreatine depletion and muscle acidification were greater in both patient groups. After exercise, the recovery of phosphocreatine-an index of oxidative metabolic capacity of the muscle-was slower in the $\beta$ myosin heavy chain group (mean half time $0.65(0.08)$ minutes) than in the troponin $T$ group $(0.60(0.17) \mathrm{min}-$ utes) or controls $(0 \cdot 48(0 \cdot 14)$ minutes $)$.

Conclusions-Exercise metabolism was abnormal in both groups of subjects, and the affected contractile protein determined the metabolic changes in muscle at rest and during recovery. In patients with abnormal $\beta$ myosin heavy chain protein, there was a decrease in oxidative capacity consistent with the reduction in mitochondria reported in muscle biopsy studies of similar patients.
\end{abstract}

(Heart 1997;78:177-181)

Keywords: hypertrophic cardiomyopathy; magnetic resonance spectroscopy; metabolism; skeletal muscle

Familial hypertrophic cardiomyopathy is characterised by asymmetrical cardiac hypertrophy, cardiac arrhythmias and sudden death. ${ }^{1}$ Contractile proteins in the cardiac muscle sarcomere (for example, $\beta$ myosin heavy chain and troponin $\mathrm{T}$ ) are abnormal in over $50 \%$ of sufferers of the disease ${ }^{23}$ although there may be variable expression of the mutant gene.
Histological studies report gross disorganisation of the cardiac myocyte architecture in familial hypertrophic cardiomyopathy ${ }^{4}$ and inefficiency in the contraction of the sarcomere may be responsible for the cardiac hypertrophy. The aetiology of the hypertrophy is not well understood since the maximum left ventricular wall thickness can differ markedly in patients with identical amino acid substitutions. ${ }^{5}$

Abnormal contractile protein has been identified in the sarcomere of skeletal as well as cardiac muscle of some but not all subjects with familial hypertrophic cardiomyopathy. ${ }^{6}$ Abnormal $\beta$ myosin heavy chain causes disorganisation of the skeletal muscle cell structure, reduced motility of the actin-myosin contractile apparatus, ${ }^{6}$ and decreased contractility of muscle fibres that is dependent upon the location of the amino acid substitution. ${ }^{7}$ In heart failure, troponin $T$ isoforms are altered in cardiac muscle but the troponin $\mathrm{T}$ isoforms that normally occur in the adult heart are not usually expressed in skeletal muscle. ${ }^{8}$ Alteration of troponin $\mathrm{T}$ in skeletal muscle in heart failure or in familial hypertrophic cardiomyopathy has not been investigated.

The effect on skeletal muscle metabolism of familial hypertrophic cardiomyopathy resulting from abnormal $\beta$ myosin heavy chain or troponin $\mathrm{T}$ was studied non-invasively by ${ }^{31} \mathrm{P}$ magnetic resonance spectroscopy. This technique assesses oxidative and non-oxidative ATP synthesis in skeletal muscle in vivo at rest, during exercise, and after recovery. These studies were designed to evaluate the effect of familial hypertrophic cardiomyopathy on phosphate containing metabolite ratios in skeletal muscle at rest, on the mechanism of ATP synthesis during exercise, and on the rate of oxidative ATP synthesis during recovery from exercise. Data were considered in the light of genetic, physiological, and clinical information.

\section{Methods}

SUBJECTS

Five patients with abnormal $\beta$ myosin heavy chain (mean age 26.0 (SD $7 \cdot 2$ ) years) and five patients with a troponin $\mathrm{T}$ abnormality (mean age $29.6(6.2)$ years) were recruited. The study had local ethics committee approval and informed consent was obtained from all subjects. All patients had a ${ }^{31} \mathrm{P}$ magnetic resonance spectroscopy study of the right leg. Recent echocardiographic and physiological exercise testing data, as well as the genetic 
data indicating the type and location of the amino acid substitution, were used to characterise the patients. Clinical information on each patient, including medication, signs and symptoms of cardiac failure, and family history of sudden death, was also collected. Some patient characteristics including the location and the type of amino acid substitution are presented in table 1 . No patient was clinically in heart failure. Eight patients were NYHA class 1 and two patients (troponin $T$ ) were NYHA class 2.

Five of the patients were on antiarrhythmic drugs; three patients were taking amiodarone (one troponin $\mathrm{T}$ and two $\beta$ myosin heavy chain), another patient (troponin T) was being treated with verapamil and a $\beta$ blocker, and another patient ( $\beta$ myosin heavy chain) was taking diltiazem. The magnetic resonance spectroscopy data were compared with results from studies of 16 activity matched normal healthy subjects of similar age (mean age $28 \cdot 2$ $(4 \cdot 1)$ years) and sex distribution.

STUDY PROTOCOL

${ }^{31} P$ magnetic resonance spectroscopy methods

Subjects lay in a $2.0 \mathrm{~T}$ superconducting magnet (Oxford Magnet Technology, Eynsham, Oxford, United Kingdom) interfaced to a Bruker spectrometer (Bruker, Coventry, United Kingdom), with the right calf overlying a $6 \mathrm{~cm}$ diameter surface coil. Spectra were acquired using a $2 \mathrm{~s}$ interpulse delay at rest (64 scans) and during exercise and recovery. The muscle was exercised by plantar flexion of the right ankle, lifting a weight of $10 \%$ lean body mass (determined from skinfold thickness by reference to standard tables) ${ }^{9}$ a distance of 7 $\mathrm{cm}$ at a rate of $30 / \mathrm{min}$. After four 32 scan spectra had been acquired (each 1.25 minutes), the weight was incremented by $2 \%$ of lean body mass for each subsequent spectral acquisition ( 1.25 minutes). The subjects exercised until stopped by fatigue. The muscle was then studied for 13 minutes during recovery (four 8 scan spectra, four 16 scan spectra, three 32 scan spectra, and two 64 scan spectra). Relative concentrations of inorganic phosphate, phosphocreatine, and ATP were obtained by a time domain fitting routine (VARPRO, $R$ de Beer, Delft, The Netherlands) and were corrected for magnetic saturation. Intracellular $\mathrm{pH}$ was calculated from the chemical shift of the inorganic phosphate peak relative to phosphocreatine. ${ }^{10}$

The data from ${ }^{31} \mathrm{P}$ magnetic resonance spec-

Table 1 Selected patient information

\begin{tabular}{lllll}
\hline $\begin{array}{l}\text { Patient } \\
\text { number }\end{array}$ & $\begin{array}{l}\text { Age } \\
\text { (years) }\end{array}$ & Sex & Abnormal protein & Mutation \\
\hline 1 & 30 & Male & $\beta$ MHC & VAL606MET \\
2 & 23 & Male & $\beta$ MHC & VAL606MET \\
3 & 40 & Male & $\beta$ MHC & GLU949LYS \\
4 & 26 & Male & M MHC & ARG453CYS \\
5 & 29 & Male & Troponin T & ARG453CYS \\
6 & 33 & Female & Troponin T & ARG92GLU \\
7 & 31 & Female & Troponin T & ARG92GLU \\
8 & 30 & Male & Troponin T & ILE79ASU \\
9 & 16 & Male & Troponin T & dGLU160 \\
\hline
\end{tabular}

ARG, arginine; ASN, asparagine; CYS, cysteine; GLU, glutamine; ILE, isoleucine; LYS, lysine; MET, methionine; MHC, myosin heavy chain; VAL, valine. troscopy were used to assess the metabolic potential of the muscle and the ability of the muscle to synthesise ATP aerobically and anaerobically. Absolute concentrations of phosphocreatine, inorganic phosphate, and ATP cannot be measured accurately using a surface coil and the concentrations of phosphocreatine and inorganic phosphate are usually expressed relative to [ATP] or as inorganic phosphate/phosphocreatine. Movement of the leg away from the coil during exercise can simulate loss of phosphocreatine from the muscle when the spectrum is analysed. The sum, phosphocreatine + inorganic phosphate, remains constant during exercise so expression of phosphocreatine changes during exercise as phosphocreatine/(phosphocreatine + inorganic phosphate) is a convenient means of accounting for signal loss due to this muscle movement. Changes in phosphocreatine/ (phosphocreatine + inorganic phosphate) and pH during exercise can reveal alterations in non-oxidative ATP synthesis. During recovery, ATP synthesis is purely oxidative, signal loss due to leg movement is not a problem, and the rate of phosphocreatine resynthesis (expressed as the recovery half time) gives an idea of the relative mitochondrial capacity of the muscle in vivo. ${ }^{1011}$

\section{DATA ANALYSIS}

Data from the three groups ( $\beta$ myosin heavy chain or troponin $\mathrm{T}$ patients and controls) were compared using either the MannWhitney $U$ test or repeated measures analysis of variance where appropriate. All patients performed at least five minutes of exercise (four spectra). Changes in phosphocreatine and $\mathrm{pH}$ from rest to the end of five minutes (five spectra in total) were analysed in each of the three groups, and each group was compared with the other two using repeated measures analysis of variance. The data from all patients during the fourth exercise spectrum were also compared using the Mann-Whitney $\mathrm{U}$ test. Time dependent comparison of exercise data after this point was affected by the different exercise duration of the individual subjects. Data are expressed as mean (SD). $\mathbf{P}<$ 0.05 was taken to indicate a significant difference.

\section{Results}

RESTING MUSCLE

The resting metabolite ratios were markedly different in the two familial hypertrophic cardiomyopathy groups studied (table 2). The group with abnormal troponin $\mathrm{T}$ was similar to controls but the abnormal $\beta$ myosin heavy chain group had a higher [phosphocreatine]/[ATP] and a lower [inorganic phosphate]/[phosphocreatine] ratio than either the abnormal troponin $T$ group or the control group. The group with abnormal $\beta$ myosin heavy chain also had a higher [inorganic phosphate]/[ATP] than controls. Muscle [ATP] and [creatine] were not quantified so the full implications of these altered ratios cannot be explored. It is possible there was an alteration 
in [ATP] and/or [creatine] in the muscle with abnormal $\beta$ myosin heavy chain which contributed to these altered ratios. As ADP concentration can only be calculated on the basis of assumed [ATP] and [creatine], [ADP] calculation was doubtful in these patients. In the light of these marked discrepancies in the resting muscle spectra of the two different groups of cardiomyopathy patients, it would be inappropriate to combine the two groups into one for analysis of the condition of familial hypertrophic cardiomyopathy as a whole.

\section{EXERCISING MUSCLE}

Exercise duration was significantly reduced in both cardiomyopathy groups $(7 \cdot 3(1 \cdot 9)$ and $8.5(3 \cdot 1)$ minutes in the groups with abnormal troponin $\mathrm{T}$ and $\beta$ myosin heavy chain respectively) compared to controls (11.9 (3.1) minutes, $P<0.05)$, but the end exercise phosphocreatine/(phosphocreatine + inorganic phosphate) was similar in all three groups (table 2). End exercise $\mathrm{pH}$ was not significantly different in the group with abnormal troponin $\mathrm{T}$ and was lower in the abnormal $\beta$ myosin heavy chain group when compared to controls.

When results over the first five minutes of exercise were examined (that is, that period during which all subjects were able to exercise), differences from controls were seen in the patients with abnormal $\beta$ myosin heavy chain, although differences from controls in the abnormal troponin $T$ patient group did not achieve statistical significance $(P=0.05$ in $\beta$ myosin heavy chain patients and $\mathrm{P}=$ 0.08 in troponin $T$ patients for changes in phosphocreatine/(phosphocreatine + inorganic phosphate); $P=0.01$ and $P=0.29$ respectively for changes in cytosolic $\mathrm{pH}$ (analysis of variance)). The fourth exercise spectrum was the last spectral acquisition during which all subjects were exercising, and during that period there were significant differences in phosphocreatine/(phosphocreatine + inorganic phosphate) and $\mathrm{pH}$ in both cardiomyopathy groups compared to controls (table 2).

\section{MUSCLE RECOVERING FROM EXERCISE}

The half time of phosphocreatine resynthesis was significantly longer in the group with abnormal $\beta$ myosin heavy chain but normal in the troponin $\mathrm{T}$ group. $\mathrm{pH}$ recovery following

Table $2{ }^{31} P$ magnetic resonance spectroscopy data, mean (SD)

\begin{tabular}{|c|c|c|c|}
\hline & Troponin $T$ & $\beta M H C$ & Control \\
\hline \multicolumn{4}{|l|}{ Resting muscle } \\
\hline [PCr]/[ATP] & $3.88(0.42)^{\star}$ & $4.51(0.17) \dagger$ & $4.04(0.40)$ \\
\hline$[\mathrm{Pi}] /[\mathrm{ATP}]$ & $0.55(0.14)$ & $0.57(0.09) \dagger$ & $0.46(0.10)$ \\
\hline$[\mathrm{Pi}] /[\mathrm{PCr}]$ & $0.11(0.02)$ & $0.10(0.01) f$ & $0.12(0.02)$ \\
\hline $\mathrm{PCr} /(\mathrm{PCr}+\mathrm{Pi})$ & $0.88(0.02)$ & $0.89(0.02)$ & $0.90(0.02)$ \\
\hline $\mathrm{pH}$ & $7.02(0.03)$ & $7.03(0.02)$ & $7.03(0.02)$ \\
\hline \multicolumn{4}{|l|}{4 min exercise } \\
\hline $\mathrm{PCr} /(\mathbf{P C r}+\mathrm{Pi})$ & $0.49(0.15) \dagger$ & $0.52(0.17) \dagger$ & $0.68(0.13)$ \\
\hline $\mathrm{pH}$ & $6.82(0.17) \dagger$ & $6.72(0.24) \dagger$ & $6.97(0.11)$ \\
\hline \multicolumn{4}{|l|}{ End exercise } \\
\hline $\begin{array}{l}\mathrm{PCr} /(\mathbf{P C r}+\mathbf{P i}) \\
\mathrm{pH}\end{array}$ & $\begin{array}{l}0.36(0.06) \\
6.69(0.13)\end{array}$ & $\begin{array}{l}0.35(0.11) \\
6.56(0.11) t\end{array}$ & $\begin{array}{l}0.38(0.11) \\
6.73(0.13)\end{array}$ \\
\hline \multicolumn{4}{|l|}{ Recovery } \\
\hline PCr resynthesis half time (min) & $0.60(0.17)$ & $0.65(0.08) \dagger$ & $0.48(0.14)$ \\
\hline
\end{tabular}

${ }_{\star P} \mathrm{P}<0.05 v \beta$ MHC group; Mann-Whitney $U$ test.

†P $<0.05 v$ controls; Mann-Whitney U test.
ATP, adenosine triphosphate; MHC, myosin heavy chain; $\mathrm{PCr}$, phosphocreatine; $\mathrm{Pi}$, inorganic phosphate. exercise was similar to control subjects in both cardiomyopathy groups (data not shown), suggesting that reduced proton efflux from the muscle was not responsible for the $\mathrm{pH}$ changes during exercise. The percentage of predicted $\dot{\mathrm{VO}} \mathrm{O}_{2}$ max was similar in both groups of cardiomyopathy subjects, indicating that the level of activity of the subject was not responsible for these changes in recovery metabolism.

The data from individual patients, when viewed separately from the cardiomyopathy group as a whole or the relevant subgroup, did not suggest a significant effect of antiarrhythmic treatment or of the location of the substitution.

DATA NOT DERIVED FROM ${ }^{31} P$ MAGNETIC RESONANCE SPECTROSCOPY

The patients with abnormal troponin $\mathrm{T}$ had a mean maximum left ventricular wall thickness of $19.6(6.9) \mathrm{mm}$ and a predicted $\mathrm{VO}_{2} \mathrm{max}$ of 71(21)\%; those with abnormal $\beta$ myosin heavy chain had a mean maximum left ventricular wall thickness of $19.0(4 \cdot 2) \mathrm{mm}$ and a predicted $\dot{\mathrm{V}} \mathrm{O}_{2} \mathrm{max}$ of $74(11) \%$. There was no relation between the extent of the metabolic abnormalities in the skeletal muscle and NYHA class, maximum left ventricular wall thickness, prognosis, location and type of amino acid substitution, contractile protein affected, or percentage of predicted $\mathrm{VO}_{2} \max$ measured on an exercise treadmill test.

\section{Discussion}

RESTING MUSCLE

Skeletal muscle manifestations of familial hypertrophic cardiomyopathy depend upon the contractile protein affected. Patients with abnormal $\beta$ myosin heavy chain protein had a significantly higher resting [phosphocreatine]/[ATP] ratio and lower [inorganic phosphate]/[phosphocreatine] ratio in skeletal muscle. Slow twitch and fast twitch fibres have slightly different [phosphocreatine]/[ATP] ratios. ${ }^{12}$ These metabolic alterations could be explained by a change in the proportions of fibre types in the calf muscles ${ }^{12}{ }^{13}$ favouring fast twitch fibres over slow twitch fibres. Muscle biopsies were not performed on these patients because two other studies have already examined muscle histology in familial hypertrophic cardiomyopathy. ${ }^{14}{ }^{15}$ Abnormal $\beta$ myosin heavy chain is expressed in the slow twitch fibres of skeletal muscle. ${ }^{6714}$ Slow twitch fibres of some patients with familial hypertrophic cardiomyopathy have central areas of marked myofibrillar degeneration and mitochondrial loss. ${ }^{15} \mathrm{~A}$ more recent study shows these central cores of degeneration are a feature of skeletal muscle biopsies of patients with abnormal $\beta$ myosin heavy chain. ${ }^{14}$ Our finding in resting muscle of those patients with abnormal $\beta$ myosin heavy chain support these other two studies. Any reduction in the relative proportion of slow to fast twitch (non-oxidative) muscle fibres in these subjects might also result in a reduced oxidative capacity (see below). The group with abnormal troponin $\mathrm{T}$ had resting muscle values similar to controls, suggesting normal fibre type distribution. 
EXERCISING MUSCLE

Both groups of patients had an altered response to exercise. When the data were analysed using repeated measures analysis of variance from rest to the fifth minute of exercise, there was a significant fall in $\mathrm{pH}$ and phosphocreatine/(phosphocreatine + inorganic phosphate) compared to normal. These changes represent an increase in non-oxidative ATP synthesis during exercise. This could reflect a central effect on skeletal muscle metabolism of cardiac hypertrophy.

A preliminary ${ }^{31} \mathrm{P}$ magnetic resonance spectroscopy study of five other patients with a $\beta$ myosin heavy chain defect has shown altered high energy phosphate metabolism during exercise and recovery, consistent with diminished ATP synthesis or inefficient ATP utilisation by myosin. ${ }^{16}$ In the present study, the increased changes in phosphocreatine and $\mathrm{pH}$ during exercise in the cardiomyopathy patients reflect an increased demand for ATP synthesis, suggesting that inefficient ATP utilisation is more likely. Actinomyosin interactions within the skeletal muscle of patients with abnormal $\beta$ myosin heavy chain are abnormal since mutant $\beta$ myosin translocates actin filaments more slowly than controls ${ }^{6}$ and contractility is lower in muscle fibres containing certain abnormal forms of $\beta$ myosin heavy chain. ${ }^{7}$ Increased demand for ATP to provide energy for muscle work would result in increased ATP synthesis to maintain normal contraction during exercise and this would manifest itself as the changes noted by our investigation. Studies in vitro have not yet clarified whether increased ATP consumption does occur in working skeletal muscle in familial hypertrophic cardiomyopathy.

Physical deconditioning due to inactivity is unlikely to be responsible for these changes. We used an activity matched group of controls, and another study has shown that patients with chronic fatigue syndrome and a history of relatively little daily muscle activity had normal results from ${ }^{31} \mathrm{P}$ magnetic resonance spectroscopy studies of their muscles. ${ }^{17}$ Moreover, the differences seen in the metabolism of these patients are unlikely to be due to antiarrhythmic drug treatment. In other published reports and in our considerable experience with ${ }^{31} \mathrm{P}$ magnetic resonance spectroscopy, none of these drugs has been reported to cause metabolic defects that could be detected by ${ }^{31} \mathrm{P}$ magnetic resonance spectroscopy of skeletal muscle.

There has been no study examining the troponin $\mathrm{T}$ expression in skeletal muscle of patients with abnormal cardiac troponin $T$. Therefore it is difficult at present to explain the metabolic changes and reduced exercise duration in the troponin $T$ group. Heart failure is an unlikely reason since it causes abnormalities in recovery from exercise. Despite two patients with NYHA class 2 symptoms being included in the abnormal troponin $\mathrm{T}$ group, there were no recovery abnormalities compared to the control group nor were there abnormalities within the abnormal troponin $\mathrm{T}$ group attributable to these two patients.
MUSCLE RECOVERING FROM EXERCISE

Following exercise, the recovering muscle resynthesises phosphocreatine aerobically and the rate of this resynthesis yields a measure of the capacity of the muscle for aerobic ATP synthesis. ${ }^{10}$ The aerobic capacity was assessed by the half time of phosphocreatine recovery, a reasonable assessment of relative mitochondrial capacity if end exercise [phosphocreatine] is similar in the groups being compared. ${ }^{11}$ Compared to normal muscle, the skeletal muscle of patients with familial hypertrophic cardiomyopathy due to abnormal $\beta$ myosin heavy chain had a slower recovery of phosphocreatine following exercise. Soleus muscle biopsies of patients with abnormal skeletal muscle expression of $\beta$ myosin heavy chain show reduced numbers of mitochondria $^{14}$ and this would contribute to the reduced oxidative capacity seen in these patients. The group with abnormal troponin $T$ had no abnormality in recovery from exercise.

\section{DATA NOT DERIVED FROM ${ }^{31} P$ MAGNETIC}

\section{RESONANCE SPECTROSCOPY}

The location of the amino acid substitution in subjects with abnormal $\beta$ myosin heavy chain appeared to influence the contractility of isolated muscle fibres. ${ }^{7}$ The number of patients we studied precluded a case by case correlation of metabolic defects with substitution site. We saw no relation between the echocardiographic or exercise treadmill testing data and any of the ${ }^{31} \mathrm{P}$ magnetic resonance spectroscopy findings, reinforcing other studies that have failed to correlate skeletal muscle and cardiac manifestations of familial hypertrophic cardiomyopathy. ${ }^{1141518}$

\section{SUMMARY}

Using ${ }^{31} \mathrm{P}$ magnetic resonance spectroscopy, we have discovered exercise induced abnormalities in skeletal muscle metabolism of patients with familial hypertrophic cardiomyopathy. These metabolic changes could contribute to the exercise intolerance of patients with this condition. Skeletal muscle metabolism in familial cardiac hypertrophy depends upon the contractile protein affected. In a group of patients where the condition was caused by abnormal $\beta$ myosin heavy chain protein, there were additional metabolic changes seen at rest and during recovery from exercise. The changes in recovery imply a decrease in the oxidative capacity of the muscle, which is consistent with reports of a reduction in the number of mitochondria in the slow twitch fibres in the leg muscle of these patients.

This work was funded by the British Heart Foundation and the Medical Research Council of Great Britain.

\footnotetext{
1 Maron B, Bonow R, Cannon R, Leon M, Epstein S. Hypertrophic cardiomyopathy: interrelations of clinical manifestations, pathophysiology, and therapy. $N$ Engl $\mathcal{f}$ Med 1987;316:780-9, 844-52.

2 Watkins H, Rosenzweig A, Hwang D-S, Levi T, McKenna W, Seidmann CE, et al. Characteristics and prognostic in familial hypertrophic cardiomyopathy. N Engl f Med 1992;326:

3 Watkins H, McKenna W, Thierfelder L, Suk HJ, Anan R, O'Donoghue A, et al. Mutations in the genes for cardiac troponin $\mathrm{T}$ and $\alpha$ tropomyosin in hypertrophic cardiomy-
} 
opathy. N Engl F Med 1995;332:1058-64

4 Sutton M, Lie J, Anderson K, O'Brien P, Frye R. Histopathological specificity of hypertrophic obstructive cardiomyopathy. Br Hear $\mathcal{f} 1980 ; 44: 433-43$.

5 Anan R, Greve G, Thierfelder L, Watkins H, McKenna WJ, Solomon S, et al. Prognostic implications of novel $\beta$ cardiac myosin heavy chain mutations that cause familial hypertrophic cardiomyopathy. F Clin Invest 1994;93: 280-5.

6 Cuda G, Fananapazir L, Zhu W-S, Sellers J, Epstein N. Skeletal muscle expression and abnormal function of myosin in hypertrophic cardiomyopathy. $f$ Clin Invest myosin in hype

7 Lankford E, Epstein N, Fananapazir L, Sweeney H. Abnormal contractile properties of muscle fibers expressing $\beta$-myosin heavy chain gene mutations in patients with hypertrophic cardiomyopathy. F Clin Invest 1995;95: 1409-14.

8 Anderson P, Malouf N, Oakeley A, Pagani E, Allen P. Troponin $T$ isoform expression in humans. Circ Res 1991;69:1226-33.

9 Durnin J, Womersley J. Body fat assessed from total body density and its estimation from skinfold thickness; measurements in 481 men and women aged from 16 to 72 years. Br 7 Nutr 1974;32:77-97.

10 Arnold DL, Matthews PM, Radda GK. Metabolic recovery after exercise and the assessment of mitochondrial function in vivo in human skeletal muscle by means of P-31 NMR. Magn Reson Med 1984;1:307-15.

11 Kemp GJ, Thompson CH, Taylor DJ, Hands LJ,
Rajagopalan B, Radda GK. Quantitative analysis by ${ }^{31} \mathrm{P}$ MRS of abnormal mitochondrial oxidation in skeletal muscle during recovery from exercise. NMR Biomed 1993;6:302-10

12 Meyer R, Brown T, Kushmerick $M$. Phosphorus nuclear magnetic resonance of fast- and slow-twitch muscle. $A m \mathcal{F}$ Physiol 1985;248:C279-87.

13 Vandenborne K, Walter G, Ploutz-Snyder L, Staron R, Fry $A$, de Meirleir K, et al. Energy-rich phosphates in slow and fast human skeletal muscle. Am f Physiol 1995; 268:C869-76.

14 Fananapazir L Dalakas M, Cyran F, Cohn G, Epstein N. Missense mutations in the $\beta$ myosin heavy-chain gene Missense mutations in the $\beta$ myosin heavy-chain gene cause central core disease in hypertroph

15 Smith ER, Heffernan LP, Sangalang VE, Vaughan LM, Flemington CS. Voluntary muscle involvement in hypertrophic cardiomyopathy. Ann Intern Med 1976;85: 566-72.

16 Ryschon T, Fowler M, Arai A, Wysong RE, McAreavey D, Epstein ND, et al. Muscle energetics during dynamic exercise in hypertrophic cardiomyopathy [abstract]. Circulation 1994;90:I-442.

17 Barnes P, Taylor D, Kemp G, Radda G. Skeletal muscle bioenergetics in the chronic fatigue syndrome. 7 Neurol Neurosurg Psychiatry 1993;56:679-83.

18 Spirito P, Maron BJ, Bonow RO, Epstein SE. Severe functional limitation in patients with hypertrophic cardiotonal limitation in patients with hypertrophic cardiohypertrophy. $7 \mathrm{Am}$ Coll Cardiol 1986;8:537-44. 